\title{
DERECHOS HUMANOS DE LA NIÑEZ: CAMBIO DE PERSPECTIVA Y DESAFÍO EDUCATIVO
}

Childrens rights: changing perspective and education challenge

Direitos humanos da criança: mudança de perspectiva e desafio educativo

\section{Álvaro Sepúlveda Romero}

Fundación Marista de Solidaridad Internacional, Chile. Fono: +56 26890880. Correo electrónico:

asepulveda@maristas.cl

\section{Resumen}

La finalidad de este artículo es profundizar en la tensión que implica, por una parte, el derecho que tienen los niños, niñas y adolescentes a opinar en todo lo que les afecte y ejercer un rol activo en su sociedad y, por otra, el derecho a ser protegidos por parte de los adultos, de un modo respetuoso. Protección que, sin embargo, muchas veces corre el riesgo de expresar la imposición adultocéntrica sobre el grupo social que constituye la infancia, a quienes se les relega mediante prácticas culturales y decisiones políticas. Muestra de ello ha sido la sistemática reticencia a armonizar la legislación nacional de acuerdo a la Convención sobre los Derechos del Niño, ratificada por el Estado Chileno hace veinticinco años. Finalmente, se reflexiona sobre las implicancias que tiene este nuevo enfoque para la educación y las oportunidades que se vislumbran para la escuela ante el nuevo escenario.

Palabras clave: Enfoque de derechos, participación infanto juvenil, ejercicio de derechos.

\begin{abstract}
The purpose of this article is to deepen the tension that implies, on the one hand, the right of children and young people to give an opinion in everything that affects them and to play an active role in society and, in the other hand, the right to be protected by adults in a respectful way. However, very often the risk is that adults, on pretending provide protection, imposse their perspectives on children and relegate them, as a social group, by cultural practices and policy decisions. Proof of this is the systematic reluctance to harmonize national legislation in accordance with the Convention on the Rights of the Child, ratified by the Chilean State twenty-five years ago. Finally, we
\end{abstract}


examine the implications for this new approach to education when new opportunities are emerging for the school in the new scenario.

Keywords: Human rights approach, Children's and youth participation, Exercise human rights.

\section{Resumo}

A finalidade deste artigo é aprofundar a tensão que envolve, de um lado, os direitos das crianças e adolescentes de opinarem em tudo o que os afete e seu papel ativo na sociedade e, por outro lado, os direitos de serem protegidos pelos adultos de maneira respeitosa. Proteção que, sem dúvida, muitas vezes corre o risco de expressar a imposição adultocêntrica sobreo o grupo social que constituí a infância, as quais se vê mediante práticas culturais e decisões políticas. Uma demonstração disso é o posicionamento reticente sobre harmonizar a legislação nacional em consonância a Convenção da Criança, ratificada pelo Estado chileno há vinte e cinco anos. Finalmente, se reflete sobre as implicações que este novo enfoque para a educação e as oportunidades que se vislumbram para a escola diante de um novo cenário.

Palavras-chave: Enfoque de direitos, participação infanto juvenil, exercício de direitos.

"Por miedo a que la muerte nos pueda arrebatar al niño,
nosotros lo privamos de la vida;
a fin de evitar su muerte,
no permitimos que viva en plenitud"
Janusz Korczak

\section{Introducción}

En temas como los derechos de los niños, las representaciones sociales más cotidianas se conjugan con las políticas de Estado regresivas para entorpecer la garantía plena de derechos.

No siempre resulta fácil traspasar las barreras culturales que mantienen a la primera infancia sujeta a los estereotipos de una ternura cosificante, a la niñez encerrada en la idea de una inocencia acrítica y a los adolescentes sujetos a la imagen mercantilizada de la diversión sin fin. Quienes provienen de sectores empobrecidos, además, son percibidos como objetos de compasión y cuidado o, en el peor de los casos, como factores de riesgo para la seguridad y el orden público. 
En primera instancia, nadie sería capaz de oponerse a iniciativas que favorecen a la población de entre 0 y 18 años. Sin embargo, el hecho de que Chile sea el único país de América Latina que aún no cuenta con la debida adecuación legislativa en esta materia, demuestra que dicha ternura, inocencia, compasión y cuidado son, de algún modo, expresiones de un modelo de atención meramente tutelar y manifestación de las ataduras que se rehúsan al reconocimiento de niños, niñas y adolescentes como sujetos plenos de derechos.

El adultocentrismo imperante mantiene a la niñez retenida en una 'jaula con barrotes dorados'. Pareciera ser que existe toda la disposición para darles los cuidados que requiere su bienestar; incluso el mercado los convierte en argumento para estimular el consumo en determinadas fechas. Pero esta supuesta atención prioritaria no implica una escucha atenta y respetuosa de sus opiniones. Mucho menos, tomarlos en cuenta al momento de decidir en ámbitos donde ellos y ellas se ven afectados.

La patologización del comportamiento infantil y su consecuente medicalización, el desbalance etario del bienestar, la naturalización del castigo corporal, son sólo algunos de los indicadores que muestran la desventaja en que se hallan los niños, niñas y adolescentes. Dejan en evidencia las reticencias aún existentes para aceptar que este inmenso grupo de personas (más de un cuarto de la población nacional), son seres humanos que merecen ser tratados sin ningún tipo de discriminación. Son titulares de derechos civiles y políticos y, por ende, pueden informarse, pensar, creer y opinar con libertad, asociarse, reunirse y ser tomados en cuenta.

Es en este contexto que el sistema educacional tiene la oportunidad de cuestionar el rol que tradicionalmente se le ha asignado, como espacio de socialización, adoctrinamiento y asimilación, para asumir el desafío de ser una instancia donde los niños, niñas y adolescentes pueden experimentar lo que significa tener derechos $\mathrm{y}$ aprender a ejercerlos con responsabilidad. Al mismo tiempo que desarrollar la capacidad de convivir con otros en un espíritu de respeto, tolerancia y solidaridad.

\section{El difícil tránsito del riesgo social al enfoque de derechos}

La Ley de Menores promulgada durante el gobierno del presidente Eduardo Frei Montalva, en 1967, significó un avance sustantivo en la regularización de la protección 
de niños en situación de riesgo social. Una publicación de la época reconocía sus beneficios para solucionar el problema de los 'irregulares sociales' o los 'niños de la calle'. Para ellos existían distintas instituciones que, sin embargo, lograban atender apenas un $10 \%$ del total de los casos.

Estas organizaciones operaban bajo distintos reglamentos hasta el 8 de marzo de 1967, cuando se promulgó la ley que creó el Consejo Nacional de Menores (...) La misma ley creó la Policía de Menores y dio autoridad a este organismo para recoger a los niños vagos; apareció la 'mosca azul', que se convirtió en el terror de estos vagabundos de corazón; por ningún motivo querían ser llevados a los hogares: 'Chiis, ¿no ve que me llevan preso?' (...) El reformatorio es para estos niños la única medida eficaz y su reclusión debe prolongarse todo el tiempo necesario para consumar la obra de readaptación social”(Urrutia, 1972: 80)

Se trató de una legislación elaborada desde el foco de la Doctrina de la Situación Irregular, destinada a niños víctimas de la pobreza, negligencia o maltrato. Implícitamente, procuraba también prevenir el riesgo que la falta de vigilancia hacia estos niños podría significar para la sociedad.

Como es lógico, se estaba pensando en un grupo numeroso, pero acotado de niños y niñas. Grupo que se ha ido reduciendo en la medida que han mejorado las condiciones socioeconómicas del país. Desde ese entonces, Chile ha visto transitar regímenes democráticos y autoritarios, ha alcanzado metas macroeconómicas de las cuales se enorgullece, ha experimentado transformaciones culturales significativas e, incluso, fue incorporado en el selecto grupo de países que conforman la OCDE.

Por esta razón es paradójico que después de casi cincuenta años la legislación nacional que orienta los asuntos referidos a la niñez, siga siendo la misma Ley de Menores de 1967, tal como si el Estado Chileno no hubiese promulgado el 14 de agosto de 1990 la Convención sobre los Derechos del Niño.

Esta Convención es el tratado internacional ratificado por la mayor cantidad de estados, 195 hasta la fecha. Actualmente, Estados Unidos de América y Sudán del Sur son los únicos países que no han incorporado la Convención a su legislación interna.

Las observaciones generales del Comité de Derechos del Niño de Naciones Unidas (CRC, por su sigla en inglés) son herramientas que permiten ampliar la doctrina de los derechos humanos de la niñez. Hasta la fecha, el CRC ha emitido 17 observaciones generales. La número 5, formulada en 2003, se refiere a las medidas 
generales de aplicación de la Convención. Entre las obligaciones que los Estados Parte deben cumplir se encuentra 'hacer que toda la legislación interna sea plenamente compatible con la Convención y que los principios y las disposiciones de ésta puedan aplicarse directamente'. La mayoría de quienes la han ratificado, empezaron por adecuar su legislación, luego sus políticas públicas y, posteriormente, han creado instituciones específicas para la niñez. Chile aún no da el primer paso.

A pesar de los ingentes esfuerzos que ha hecho la sociedad civil, e independientemente de los gobiernos de turno, ha resultado imposible la adecuación legislativa. Incluso más, ha sido muy dificultoso hacer entender que el enfoque de derechos consagrado en la Convención garantiza los derechos de "todo ser humano menor de dieciocho años de edad" (Art. 1), sin distinción de ningún tipo, ni siquiera de su posición económica (Preámbulo). No se trata sólo de los niños de la calle o en riesgo social, por quienes velaba la clásica 'protección especial'.

Luego de veinticinco años desde que se incorporara este tratado internacional es, al menos, vergonzoso reconocer que el marco jurídico en materia de niñez y adolescencia sigue siendo una legislación pensada para

los pequeños y niñitas que, en el más crudo invierno, pululan por las calles o duermen apiñados en los umbrales de las tiendas del centro, ante la indiferencia de los transeúntes que corren presurosos a sus hogares (Urrutia, 1972: 78).

Por supuesto que los niños y niñas más pobres son motivo de particular atención, nadie pone en duda este hecho. En la actualidad se reconoce que algunos grupos como los niños y niñas migrantes (o de familias migrantes); los con capacidades diversas; los gays, lesbianas, transexuales, bisexuales e intersex (LGTBI); y los indígenas, requieren de garantías explícitas y reforzadas, dentro de un marco normativo de protección especial (UNICEF, 2015). Estos grupos se destacan por sobre el resto y son una prioridad, entendiendo que la garantía reforzada de sus derechos se sitúa dentro de la protección universal que consagra la Convención, la cual vela por la dignidad de toda persona menor de 18 años, independientemente de cualquier tipo de particularidad.

Cuando los defensores de derechos humanos hablan de los derechos de la mujer, no están pensando sólo en las mujeres más pobres o vulneradas; se están refiriendo a los derechos de la mitad de la población mundial. Así mismo, defender los 
derechos de los niños y niñas obliga a pensar y abogar por toda la población infanto adolescente.

Son estos importantes matices los que no han logrado ser comprendidos en Chile durante un cuarto de siglo. Sólo como punto de comparación, en diciembre de 2014 México promulgó la Ley General de los Derechos de Niñas, Niños y Adolescentes. Ésta vino a reemplazar la Ley para la Protección de los Derechos de Niñas, Niños y Adolescentes, del año 2000 (Castellanos y Conde, 2014). También en 2014 el Estado Plurinacional de Bolivia promulgó una nueva versión del Código Niña, Niño y Adolescente (la anterior databa de 1999). En junio de 2015 Venezuela actualizó su Ley Orgánica para la Protección de Niños, Niñas y Adolescentes (las versiones preliminares correspondían a los años 2000 y 2007). Y así se podría seguir con otra serie de países de la región y, por supuesto, de las naciones más desarrolladas que han adecuado su marco normativo a la Convención y han hecho actualizaciones, en coherencia con la evolución teórica y las nuevas demandas de la realidad.

Ni las alianzas creadas entre organismos de la sociedad civil y parlamentarios de distintas bancadas, ni las mesas de trabajo con diferentes ministerios ni la movilización de los niños y niñas se han traducido en la ansiada armonización legislativa.

En la cuenta pública de 2015, la Presidenta Bachelet asumió cuatro compromisos referidos directamente a la infancia: ampliación del Chile Crece Contigo, que cubrirá a los niños hasta que terminen $4^{\circ}$ básico; la elaboración de la Política Nacional de Niñez y Adolescencia; la creación de 80 nuevas Oficinas de Protección de Derecho; y la presentación del proyecto de ley de Garantía de Derechos de Niños y Adolescentes.

En caso de hacerse efectivo este último compromiso, sería un paso para empezar un efectivo cambio de perspectiva y para darle a todos los niños, niñas y adolescentes el reconocimiento que les corresponde, como sujetos de derechos y no sólo como objetos de protección.

Actualmente, Chile cuenta con un total de 4.423 .910 personas que se ubican entre los 0 y los 18 años. Es, entonces, a estos casi cuatro millones y medio de 
compatriotas a quienes debería amparar la Convención y la - hasta ahora inexistente ley de garantías o ley de protección integral de derechos de niños, niñas y adolescentes.

\section{De la protección a la participación}

Una de las formas en que se clasifican los derechos contenidos en la Convención, es separándolos en tres grupos: de Provisión o prestación (referidos a la satisfacción de necesidades básicas), de Protección (contra actos perjudiciales) y de Participación (el derecho a intervenir en decisiones que son de su interés). A esta clasificación conocida como 'las tres P' se le puede agregar una cuarta: la Prevención, referente a las medidas para evitar la violación de derechos y favorecer su ejercicio.

Tanto la Declaración de Derechos del Niño de 1924 (la llamada Declaración de Ginebra) como la posterior Declaración de 1959 consideraban la importancia de proveer a los niños de todo lo que requiriesen para su vida y desarrollo. Asimismo, estipulaban la necesidad de que fuesen protegidos de todo lo que afectara su bienestar. No obstante, no se hacía mención alguna a los derechos civiles y políticos. El énfasis estaba puesto en comprender a los niños desde una visión más bien tradicional, como receptores pasivos de cuidados.

Es de justicia reconocer que constituyeron un peldaño importante en el relevamiento de la dignidad de niños y niñas. Desde el siglo XIX y, en gran parte, como reacción a los efectos de la Revolución Industrial, distintos sectores crearon conciencia acerca de las vulneraciones extremas a las que estaban expuestos en el ámbito familiar y laboral. Es la época del surgimiento de las Sociedades Protectoras de la Infancia. Para subrayar la precaria sensibilidad que existía en la época, Reis (2008) destaca que esto sólo ocurrió con posterioridad a la creación de las Sociedades Protectoras de los Animales. Ejemplifica con el caso de una trabajadora social de Nueva York que en 1874 debió invocar la legislación de protección de los animales para alegar por una niña herida que encontró atada a una cama y alimentada sólo con pan y agua: como un perro o un gato, esta niña también pertenecía al reino animal y, por tanto, merecía protección.

La Protectora de la Infancia en Chile fue fundada en 1894, como respuesta a la orfandad generada por la Guerra del Pacífico. En 1895 en el Arzobispado de Santiago se erige la Congregación de las Hijas de San José Protectoras de la Infancia, como respuesta a la niñez desvalida. En 1924 se funda en Montevideo el Instituto 
Internacional Americano de Protección a la Infancia, que posteriormente ha evolucionado en el Instituto Interamericano del Niño, la Niña y Adolescentes (IIN), organismo especializado de la OEA en esta materia.

Durante la segunda mitad del siglo XIX y gran parte del XX primó un modelo de protección tutelar que procuraba proteger, especialmente, al niño en situación irregular o que padecía las consecuencias de grandes males sociales como la pobreza, las hambrunas o las guerras. Reflejo de ello son las Declaraciones de Derechos del Niño 1924 y de 1959. Este aspecto de la conciencia humanitaria hacia la niñez fue acogido, desarrollado y ratificado por la Convención de 1989.

Pero al incluir en este tratado el tercer grupo de derechos (los de Participación) se marcó el punto de inflexión con respecto a la tradición previa.

Entre los derechos de Participación se cuentan estos seis artículos de la Convención:

- Artículo 5: sobre la responsabilidad de los padres de impartirles orientación apropiada para que los niños ejerzan sus derechos.

- Artículo 12: sobre el derecho a ser escuchados y expresar su opinión libremente en todos los asuntos que les afectan.

- Artículo 13: sobre el derecho a la libertad de expresión; incluyendo la libertad de buscar, recibir y difundir informaciones e ideas de todo tipo.

- Artículo 14: sobre la libertad de pensamiento, de conciencia y de religión.

- Artículo 15: sobre la libertad de asociación y la libertad de celebrar reuniones pacíficas.

- Artículo 17: sobre el acceso a información, en especial a material que tenga por finalidad promover su bienestar social, espiritual y moral y su salud.

La Convención posiciona a los niños y niñas desde un rol activo, con capacidad para actuar y desarrollar un pensamiento propio. Fortalece su estatus social con respecto al resto del mundo adulto.

\section{La última minoría: formas actuales de dominación}


Autores como el ya citado Reis (2008), consideran que la Convención de 1989 constituyó el tercer gran hito en la historia moderna de los derechos humanos.

Luego de la Declaración de los Derechos del Hombre y del Ciudadano, al inicio de la Revolución francesa (1789), y de la Declaración Universal de los Derechos Humanos (1948), la Convención sobre los Derechos del Niño, vendría a reivindicar a los niños y niñas del lugar del que han sido históricamente relegados.

La Convención de 1989 es como la Toma de Bastilla para la liberación del último gran grupo de oprimidos de derecho y de hecho - las niñas y los niños -, paradigmas históricos de la opresión (Reis, 2008: 11).

Para comprender esta idea de la infancia como minoría, es útil recurrir a las nuevas búsquedas conceptuales que se han desarrollado desde la sociología. Entre las variantes de las nuevas sociologías de la niñez (Gaitán, 2006) la corriente estructural entiende que la infancia es una categoría social particular, que ocupa un lugar permanente en la estructura social y mantiene una interacción constante con los otros grupos etarios.

Existe intercambio permanente y relaciones de poder entre el grupo de población que forma la infancia y el resto de la sociedad adulta. Desde esta perspectiva, la niñez

es comparable y análoga, por ejemplo, a la clase o al género. Como categoría social permanente en la sociedad, sus miembros cambian, pero la infancia y sus relaciones con el grupo social mayoritario (adulto) continúan, siendo esto un componente esencial del orden social (Gaitán, 20016: 14).

Se asume que la infancia es una minoría sujeta a las tendencias de marginación y paternalización. Esta situación se manifiesta de forma particular en las sociedades modernas donde, como consecuencia de la industrialización y de las políticas de escolarización universal, han dejado al niño y al adolescente en condición de minoridad, sometidos a un proceso de formación y socialización que los hará valiosos únicamente en el futuro, cuando sean adultos.

En el marco del movimiento norteamericano por los derechos civiles de los años 70 y 80 del siglo pasado, el Movimiento por la Liberación de los Niños propugnó que, tal como las minorías étnicas y las mujeres lograban conquistas sociales, los niños 
debían ser entendidos como la 'última minoría' (Liebel, 2009). A su juicio, no existen razones válidas por las cuales los niños y niñas no pueden participar en decisiones importantes.

La invisibilidad de la infancia, de sus opiniones y de su aporte específico, viene a ser efecto directo de la dependencia a la que están sometidos.

Hoy en día los mecanismos de dominación de este grupo social adoptan solapadas maneras. Si bien ya no es la imagen grotesca de los miles de niños explotados en las minas de carbón, hoy en día el dominio de los adultos ocupa formas mucho más sofisticadas: el paternalismo extremo; el desbalance etario del bienestar; la patologización del comportamiento infantil; la naturalización de la violencia como medida de disciplina. Son manifestaciones de cómo la infancia sigue siendo una categoría social que se mantiene relegada a un segundo plano.

La encuesta CASEN 2011 indicaba que la pobreza entre los menores de 18 alcanzó a un 22,8\%. Esto significó 8,4 puntos porcentuales por sobre la cifra de pobreza nacional $(14,4 \%)$ y 11,2 puntos porcentuales más que en el segmento adulto (éste sólo llegó al 11,6\%).

El análisis resulta preocupante cuando las cifras se desagregan por criterios como el sexo, el territorio o la edad. Así, encontramos situaciones como el 38,3\% de pobreza entre los niños y niñas de 6 a 13 años de La Araucanía. La primera infancia es el grupo que acumula el mayor porcentaje de pobreza en el país, llegando a un 23,8\% (Observatorio Niñez y Adolescencia, 2013).

También es posible observar cómo mientras la pobreza nacional osciló entre un $13,7 \%$ en 2006 , un $15,1 \%$ en 2009 y un $14,6 \%$ en 2011 , la pobreza infantil aumentó progresivamente de $20,6 \%$, a $22 \%$ y $22,8 \%$ en los mismos años. En la última CASEN (2013), con la medida actualizada de pobreza por ingreso, la pobreza en el tramo de 0 a 18 años llega al 22\% (en el país es $14,4 \%$ ). Y de acuerdo a la recién inaugurada 'Pobreza Multidimensional', mientras la cifra nacional es de 20,4\%, en el caso de niños y adolescentes asciende al $23,4 \%$.

En la comprensión que Galtung (2003) tiene de la violencia, ésta asume tres formas: la violencia directa o fáctica, la violencia cultural y la violencia estructural. Es en esta última categoría donde cabe ubicar el desbalance etario del bienestar o las 
condiciones de desventaja socioeconómica en que la infancia se encuentra con respecto a los adultos.

Una práctica de violencia cultural hacia la niñez que aún está fuertemente arraigada es el castigo corporal y físico. Pocos años atrás circuló por las redes sociales con total complacencia un 'gracioso' video donde se mostraba a una madre tocando guitarra; a su lado estaban sus dos hijos de unos 6 y 8 años. En la escena se percibe gran ternura hasta que en un momento la madre se molesta por la actitud del niño y les pega a ambos sendos golpes en la cabeza. El paso abrupto del candor a la agresión resultó ser motivo de entretención masiva. Sin embargo, si en lugar de la madre hubiese estado un hombre y en vez de los niños estuviese su esposa, los graciosos golpes habrían despertado el rechazo colectivo por ser un ejemplo de violencia de género. Lo mismo si la agresión hubiese sido de una hija hacia su madre anciana o de una 'patrona' hacia su empleada. En todos esos casos, la violencia resulta indignante. Pero algo ocurre en las representaciones sociales de la infancia para que la reacción sea distinta en el caso de los niños, sobre todo si quien agrede es uno de sus progenitores.

Los resultados del informe Infancia Cuenta en Chile (Observatorio Niñez y Adolescencia, 2014) indicaron una leve disminución en la tasa de denuncia por violencia intrafamiliar en 2012 (232,1 por cada 100.000 habitantes) con respecto al año anterior. Aún así, la vulneración del derecho a ser protegido contra toda forma de maltrato en el hogar, sigue siendo un asunto que afecta principalmente a las mujeres y sobre todo a las adolescentes: $62,7 \%$ de las denuncias corresponde a las niñas; $56,3 \%$ son denuncias de adolescentes.

La segunda de las resoluciones del XXI Congreso Panamericano del Niño, la Niña y Adolescentes (Brasilia, diciembre de 2014), pide a los Estados Miembros adecuar su normativa interna, asignando presupuesto para desarrollar planes, proyectos y programas que prevengan, combatan, y eliminen la violencia, incluso el castigo corporal en todos los ámbitos. Ya son ocho los países latinoamericanos que han reformado sus leyes para prohibir el castigo físico y humillante en todas sus formas.

En el caso de Chile, el Código Penal protege a los niños contra ciertas formas de violencia, incluso en el hogar, pero no prohíbe el castigo corporal en la crianza. Tampoco lo explicita la Ley de Violencia Intrafamiliar (2005), aunque señala el deber 
del Estado de "garantizar la vida, integridad personal y seguridad de los miembros de la familia" (art. 2 y 3).

El castigo corporal se considera ilegal en los establecimientos educacionales, de acuerdo a la Ley General de Educación (2010) y a la Ley sobre la Violencia Escolar (2011). Sin embargo, en el hogar, en los centros de acogida y en los centros diurnos todavía no se ha prohibido.

El artículo 234 del Código Civil proclama el "derecho a corregir" de los progenitores. Y a pesar de que el Comité de Derechos del Niño ha solicitado modificar este artículo, ello no ha ocurrido hasta la fecha. Actualmente, Chile se encuentra en el proceso de examen por parte de dicho Comité ( $70^{\circ}$ período de sesiones) y una de las cuestiones que se le ha pedido responder se refiere precisamente a este punto.

Uno de los temas que se han comenzado a instalar en la agenda latinoamericana de infancia es la medicalización de los niños. Junto con la niñez migrante y medios de comunicación, estuvo entre los asuntos tratados en la última reunión de Iniciativa Niño@sur (Brasilia, mayo de 2015), instancia creada por la Reunión de Altas Autoridades sobre Derechos Humanos y Cancillerías del MERCOSUR, para promover la Convención y otros instrumentos de derechos humanos que atañen a los niños y niñas.

Aun cuando el Manual Diagnóstico y Estadístico de Trastornos Mentales de la Asociación Americana de Psiquiatría (DSM IV) está ampliamente difundido en el campo de la salud mental, es un instrumento cuestionado desde distintos enfoques. Para efectos del tema que nos convoca, viene a ser un ejemplo nítido de los mecanismos a través de los cuales se disciplina y somete a los niños en la actualidad. Basta con observar críticamente el capítulo destinado a los trastornos de inicio en la infancia, niñez y adolescencia.

El diagnóstico de "Déficit de Atención con Hiperactividad" contiene tres variantes: desatención, hiperactividad e impulsividad. Para corresponder a alguna de ellas, debe presentar durante 6 meses comportamientos como los siguientes:

- Desatención: no atiende a detalles o comete errores por descuido en las tareas escolares; parece no escuchar cuando se le habla; no sigue instrucciones y no finaliza tareas escolares; tiene dificultades para organizar tareas y actividades; 
renuente a un esfuerzo mental sostenido; pierde objetos necesarios para las tareas; se distrae por estímulos irrelevantes; es descuidado en las actividades diarias.

- Hiperactividad: mueve en exceso manos o pies o se remueve de su asiento; abandona su asiento en la clase; corre o salta cuando es inapropiado hacerlo; tiene dificultades para jugar o dedicarse tranquilamente a actividades de ocio; suele actuar como si tuviera un motor; habla en exceso.

- Impulsividad: precipita respuestas antes de haber sido completadas las preguntas; dificultades para guardar su turno; interrumpe o se inmiscuye en actividades de otros.

Lógicamente, un comportamiento de estas características resulta desagradable para el adulto que intenta controlar al niño en el espacio escolar o familiar. Mucho más cuando se trata de niños que están sobre estimulados como efecto de las nuevas tecnologías y del ritmo de vida del entorno. Entonces, llegar a concluir entre los adultos de la familia y los adultos especialistas que el niño padece de una psicopatología, termina siendo la consecuencia más rápida y lógica. Por supuesto, esto ahorra el cuestionamiento sobre qué tan apropiadas son las metodologías y las condiciones en que se educa al niño. $\mathrm{O}$ qué tan habilidosos son los adultos para lidiar con un ser humano que se sale de los parámetros a los que están acostumbrados.

Hace pocos meses, en Argentina, la Comisión Nacional Interministerial en Políticas de Salud Mental y Adicciones, publicó un comunicado dirigido a las comunidades educativas con "Pautas para evitar el uso inapropiado de diagnósticos, medicamentos u otros tratamientos a partir de problemáticas del ámbito escolar”. El primer punto indica que los problemas en el rendimiento o en el comportamiento escolar no equivalen necesariamente a un diagnóstico. La exclusiva falta de adecuación al comportamiento y a los objetivos esperados por la escuela o la familia, no pueden ser la única base para establecer un diagnóstico.

Estas sutiles formas de dominación conviven y se conjugan con el cuidado que entregan las familias. Al niño se le medica 'porque se le quiere' e incluso se le golpea 'por su bien', para que aprenda a corregirse. A la base de estas lógicas pervive la idea de que el hijo es una propiedad privada de los padres. Los niños 'son' de sus padres. 
Así se instala en el imaginario una representación de la infancia que calza con la idea del 'niño mascota': los padres lo cuidan en extremo, lo visten, alimentan, protegen, educan (o adiestran) y capacitan en cuanta disciplina existe porque es 'su propiedad'. Por supuesto, rara vez consideran seria y respetuosamente lo que al niño le interesa ser o hacer. La Real Academia define como mascota a la 'persona, animal o cosa que sirve de talismán, que trae buena suerte'. El concepto no se aleja tanto de lo que ocurre en algunas expresiones de la paternidad - maternidad posmoderna: niños que son recibidos como 'una bendición' que sacia vacíos existenciales y a quienes se protege hasta paralizarlos.

El problema del cuidado a los niños empieza cuando se convierte en sobreprotección. Desde una perspectiva micro política, ésta es en realidad una "máscara para ocultar prácticas paternalistas que minimizan la autonomía de los niños y que va en contra de la aplicación de sus derechos" (Franklin, 1994: 61 en: Liebel, 2009: 74).

La exagerada susceptibilidad sobre la fragilidad de los niños no hace sino debilitar aún más su posición en la sociedad. Se articula un círculo vicioso donde la atribución de incompetencia gatilla la idea de que - ante todo - los niños son necesitados de protección; entonces, el adulto actúa en su lugar, negándoles la posibilidad de participar y esto provoca el que, efectivamente, terminen siendo más vulnerables.

Liebel (2009) insiste en que la concepción del adulto como un ser fuerte y el niño como alguien débil, es fruto de una constelación cultural del poder. En ningún caso se trata de una relación natural.

Ya a comienzos de los 90, Casas (1992) alertaba sobre las representaciones sociales que la población adulta tenía de los niños y la valoración que hacían en ese entonces de la recién inaugurada Convención sobre los Derechos del Niño. Los artículos peor valorados eran los que se referían a la libertad de expresión, de pensamiento, conciencia y asociación. Como si la 'concesión' de estos derechos a los niños amenazara las seguridades adultas.

Lansdown (2005) destaca que la Convención establece por primera vez en el derecho internacional una relación directa entre el niño y el Estado. Esto viene a cuestionar la acendrada idea de que el niño es una propiedad de los padres y faculta al 
Estado para intervenir en los casos que el interés superior no esté siendo resguardado por la familia. Como indica el artículo 5, la responsabilidad de impartir dirección y orientar no es una consecuencia de lo que los niños sean una propiedad (como puede serlo en el caso de una mascota) sino que es una función de la condición de los padres, hasta que el niño desarrolle la capacidad de ejercer sus derechos.

\section{Proteger y educar para ejercer derechos y participar}

La evolución de la conciencia sobre la necesidad de proteger a los niños, es una conquista de los últimos siglos y no se puede descuidar. Sin duda alguna, proveerles de las atenciones que requieren para una vida digna, significa un gran logro cuando se toma como punto de referencia las condiciones infrahumanas en que muchos debieron crecer en el pasado.

Por otra parte, los artículos 5, 12, 13, 14, 15 y 17 de la Convención sobre los Derechos del Niño, constituyen un hito en el reconocimiento de su condición como sujetos activos y progresivamente autónomos. Les alientan a ejercer sus derechos y exigirlos en tanto son titulares de los mismos.

Es, entonces, todo un reto buscar el modo de armonizar protección y participación. En el contexto familiar, escolar y comunitario sigue siendo un desafío el cuidado respetuoso y efectivo de un sujeto pleno de derechos, durante su proceso de crecimiento y desarrollo.

El ejercicio de derechos es un asunto que debe iniciarse desde los primeros años. La Observación General $\mathrm{N}^{\circ} 7(\mathrm{CRC}, 2005)$ trata sobre la realización de los derechos del niño en la primera infancia. Entre otros aspectos, destaca la importancia de respetar las opiniones y sentimientos de los niños pequeños, como participantes activos en la promoción, protección y supervisión de sus derechos.

Lejos de la creencia mayoritaria, de que los niños pequeños son seres poco desarrollados, incapacitados para comprender, comunicarse y decidir, el CRC reafirma que también a ellos se aplica el derecho a ser oídos y expresar sus opiniones. Pide que sea practicado desde los ámbitos más cercanos: en la familia, en los espacios educativos, en la comunidad. Naturalmente, esto exige paciencia y creatividad de parte de los adultos, así como capacidad para adaptarse a las formas de comunicación preferidas por los niños pequeños. 
En estas condiciones, la paternidad - maternidad y la tarea pedagógica de los adultos resulta ser una labor altamente demandante. Exige considerar la 'evolución de las facultades del niño', entendiendo que este concepto implica el que

a medida que los niños adquieren competencias cada vez mayores, disminuye su necesidad de dirección y orientación y aumenta su capacidad de asumir responsabilidades, tomando decisiones que afectan su vida" (Lansdown, 2005: 9).

Implica desarrollar permanentemente la sensibilidad para captar la evolución del hijo o del estudiante y flexibilizar los criterios para protegerlos, de modo que al momento de cuidar se esté acogiendo el parecer de quienes son cuidados. Practicar una protección que se adecue a los intereses del niño y evite inhabilitarlo.

Implica, asimismo, tener muy claros cuáles son los fines que se persiguen con la educación. Dos artículos de la Convención hablan explícitamente sobre este derecho: el artículo 28 sobre el acceso universal a la educación y el artículo 29, acerca de los propósitos que se persigue. El CRC dedicó su primera observación general (2001) justamente a desarrollar este punto.

La meta es hasta el máximo de las capacidades del niño y el sentido es inculcar el respeto de los derechos humanos y las libertades fundamentales; el espíritu de comprensión, paz, tolerancia, igualdad de los sexos y amistad entre todos los pueblos, entre otros.

La Observación General N¹2 (2009) acerca del Derecho del Niño a ser escuchado, afirma que para la realización del derecho a la educación es fundamental que se respete el derecho del niño a ser escuchado. Además, señala:

108. Para que la enseñanza de los derechos humanos haga sentir su influencia en las motivaciones y la conducta de los niños, los derechos humanos deben practicarse en las instituciones en que el niño aprende, juega y vive junto con otros niños y adultos.

110. La participación permanente de los niños en los procesos de adopción de decisiones debe lograrse mediante, entre otras cosas, los consejos de aula, los consejos de alumnos y la representación del alumnado en los consejos y comités escolares, en que los alumnos puedan expresar libremente sus opiniones sobre la formulación y aplicación de las políticas y los códigos de conducta de la escuela.

Como en todo ámbito, debe existir una coherencia entre el mensaje declarativo que se transmite y las actitudes que se practican. Si la educación es coherente con los 
fines que le han sido encomendados y si pretende formar seres humanos para que aprendan a convivir en base al respeto, no puede sino repensar las prácticas que está albergando.

Décadas atrás, cuando el Movimiento por la Liberación de los Niños abogó por afirmar los legítimos derechos que les corresponden, instaló un fuerte discurso anti pedagógico. A su juicio, el efecto de la escuela ha sido desterrar a los niños a un recinto privado y mantenerlos sometidos a la benevolencia de los adultos. Así, sus capacidades han quedado restringidas e infravaloradas.

Liebel (2013) opina, por su parte, que los derechos de los niños han sido una creación de los adultos y donde ellos no han participado ni en la discusión ni en la redacción de los mismos. Están propuestos en un lenguaje y en una lógica que no es comprensible por los niños y, hasta que esto no ocurra, no los podrán asumir como algo propio y exigirlos. Se pregunta "¿cómo lograr que niños y niñas comprendan los derechos de la niñez como derechos subjetivos o derechos de acción?" (Liebel, 2013: 24).

Independientemente de si se comparten, o no, estas críticas, la escuela tiene hoy día la oportunidad de transformarse en un espacio donde niños y adultos aprenden a desarrollarse en un clima de respeto por la dignidad del otro. Donde todos y todas pueden contar con un espacio para expresar sus propios puntos de vista y sentir que se protege su derecho a tener y ejercer derechos.

\section{Conclusiones}

La reticencia del Estado de Chile a legislar en materia de derechos de la niñez y adolescencia, es una traba para reconocer a todas las personas menores de 18 años como sujetos políticos, con capacidad para ejercer y exigir sus derechos.

Pero aunque no se ha conseguido mediante la armonización legislativa, las nuevas generaciones de adolescentes lo han demostrado ejerciéndolo en las calles. Desde hace casi diez años, han sido catalizadores de transformaciones sociales y políticas, especialmente en el ámbito de la educación.

Ante este nuevo escenario y ante la eventual presentación de una serie de reformas legislativas e institucionales en esta materia, los adultos tienen la posibilidad 
asumir una postura distinta. Cambiar el adultocentrismo por el diálogo intergeneracional, la imposición autoritaria por la escucha respetuosa, el golpe por la palabra, el silencio complaciente por la defensa decidida.

Y, en especial, tienen la opción de transformar sus prácticas de cuidado, para que ayuden a proteger a los niños y niñas sin ahogarlos ni abandonarlos.

\section{Referencias bibliográficas}

American Psychiatric Association. (1995). Diagnosis and Statistical Manual-DSM IV. Washington, DC.: American Psychiatric Press.

Casas, F. (1992). Las representaciones sociales de las necesidades de niños y niñas, y su calidad de vida. Anuario de Psicología, 53, 27-45.

Castellanos, V., y Conde, J. (2014). Balance sobre la institucionalidad en México en materia de derechos de niñas, niños y adolescentes. a 25 años de la Convención sobre los Derechos del Niño. Revista Defensor, 12, 4-12.

CRC (2001). Observación General No 1. Párrafo 1 del artículo 29: Propósitos de la educación. Ginebra: UNICEF.

CRC (2005). Observación General $N^{\circ}$ 7. Realización de los derechos del niño en la primera infancia. Ginebra: UNICEF.

CRC (2009). Observación General $N^{\circ} 12$. El Derecho del Niño a ser escuchado. Ginebra: UNICEF.

Gaitán, L. (2006) La nueva sociología de la infancia: aportaciones de una mirada distinta. Politica y Sociedad, 43, 1, 9-26.

Galtung, J. (2003). Tras la violencia, 3R: reconstrucción, reconciliación, resolución. Afrontando los efectos visibles e invisibles de la guerra y la violencia. Gernika: Bakeaz / Gernika Gogoratuz. Centro de Investigación por la Paz. España.

Lansdown, G. (2005) La evolución de las facultades del niño. Innocenti Research Institute. Ginebra: UNICEF.

Liebel, M. y Martínez, M. (Eds.). (2009). Infancia y derechos humanos: hacia una ciudadanía participante y protagónica. Lima: IFEJANT.

Liebel, M. (2013). Niñez y justicia social: repensando sus derechos. Pehuén: Santiago de Chile. 
Observatorio Niñez y Adolescencia. (2013). Violencia contra niños, niñas y adolescentes. Santiago de Chile: Observatorio Niñoz y Adolescencia.

UNICEF (2015). Igualdad y no discriminación de niños, niñas y adolescentes: necesidad de un sistema de garantías reforzadas. Santiago de Chile: UNICEF. Urrutia, C. (1972). Niños de Chile. Santiago de Chile: Ed. Quimantú. 